\section{Sharp-Device Injuries and Perceived Risk of Infection With Bloodborne Pathogens Among Healthcare Workers in Rural Kenya}

To the Editor-Healthcare workers (HCWs) worldwide face the risk of occupational infection by bloodborne pathogens, including human immunodeficiency virus (HIV), hepatitis B virus (HBV), and hepatitis $\mathrm{C}$ virus (HCV). Guidelines exist for preventing injuries from sharp devices (hereafter, sharps), as well as for postexposure evaluation and prophylaxis, ${ }^{1,2}$ but HCWs working in limited-resource settings may not have adequate access to these recommended safety measures. This is especially of concern where the prevalence of bloodborne pathogens in the patient population may be relatively high. ${ }^{3}$

To guide interventions, in 2004 we conducted an anonymous, convenience-sample survey regarding occupational exposures to bloodborne pathogens among HCWs in Maua Methodist Hospital, a 250-bed rural mission hospital located on the eastern slopes of Mount Kenya. Of the estimated 320 employees, 200 were involved in direct patient care-the majority of these were nurses. Fifty-three $(44.1 \%)$ of the 120 questionnaires distributed by unit managers were completed. Thirty-eight $(71.7 \%)$ of the 53 who completed the survey were nurses.

Twelve $(22.6 \%)$ of the respondents recalled having at least 1 exposure to blood via percutaneous injury in the preceding year, for a total of 15 exposures. The 12 most recent sharps injuries experienced by these respondents were explored in further detail (Table), and only $4(33.3 \%)$ of 12 respondents stated that they reported their injury to their supervisor or the infection control department. Eight $(66.7 \%)$ of the injured HCWs did not know the HIV infection status of the source patient, and none knew the source patient's HBV infection status.

Concern about occupational HIV exposure was strong. Sixteen $(41 \%)$ of the 39 respondents who provided estimates perceived that more than $25 \%$ of patients were infected with HIV, and $18(47.4 \%)$ of 38 respondents perceived the risk of acquiring HIV infection from an HIV-contaminated needle stick to be greater than $10 \%$. HCWs perceived the prevalences of HBV infection and HCV infection to be lower or answered that they did not know how prevalent they were. Twentyeight $(87.5 \%)$ of 32 respondents estimated less than $11 \%$ of patients were infected with HBV, and $14(77.8 \%)$ of 18 estimated that less than $1 \%$ of patients were infected with HCV. Perception of risk from contaminated needlestick injuries involving $\mathrm{HBV}$ - or $\mathrm{HCV}$-infected blood was mixed. Fifteen $(44 \%)$ of 34 respondents believed the risk of $\mathrm{HBV}$ acquisition via a contaminated needlestick injury was greater than $25 \%$; for $\mathrm{HCV}, 11(52.4 \%)$ of 21 believed the risk was less than $1 \%$.
The availability and use of preventive measures to protect HCWs from bloodborne pathogens varied. Thirty-two (65.3\%) of 49 respondents had received at least 1 dose of $\mathrm{HBV}$ vaccine ( 1 additional HCW did not receive vaccine because the HCW had already had blood tests positive for $\mathrm{HBV}$ surface antigen); only $12(24.5 \%)$ of 49 respondents reported that they had received all 3 doses of vaccine. Forty-nine $(92.0 \%)$ of 53 respondents reported that sharps disposal containers were always available when they performed their work, and 42 $(82.3 \%)$ of 51 respondents reported that they always used them when indicated (Figure). Although $41(82.0 \%)$ of 50 respondents reported that gloves were always available, 36 $(72.0 \%)$ of these respondents reported using them at least most of the time when indicated, and $12(24.0 \%)$ reported complete adherence to guidelines for glove use.

In our survey of sharps-related injuries among HCWs in a hospital in rural Kenya, we found that almost $30 \%$ of those surveyed had sustained a blood-contaminated sharps injury in the preceding year, and concern about occupationally-acquired HIV was strong. To our knowledge, this is the first estimate regarding sharps injury exposure in HCWs in Kenya. These data are consistent with previous evidence from rural

TA B LE. Exposures to Patients' Blood and to Sharp-Device (Sharps) Injuries Among 53 Healthcare Workers (HCWs) in Kenya

\begin{tabular}{lc} 
Variable & $\begin{array}{c}\text { Proportion (\%) } \\
\text { of respondents }\end{array}$ \\
\hline $\begin{array}{l}\text { Blood expos Ire in preceding year } \\
\text { Splash to the eyes or mouth }\end{array}$ & $10 / 53(18.9)$ \\
$\quad \begin{array}{l}\text { Percutaneous or sharps injury (excluding } \\
\text { clean needles) }\end{array}$ & $15 / 53(28.3)$ \\
Most recent sharps-related blood exposure & \\
Type of sharp involved & \\
$\quad$ Solid-bore (suture) needle & $5 / 12(41.7)$ \\
$\quad$ Hollow-bore needle & $5 / 12(41.7)$ \\
$\quad$ Blood lancet & $1 / 12(8.3)$ \\
$\quad$ Scalpel or razor & $1 / 12(8.3)$ \\
Outcome & \\
$\quad$ Reported to supervisor or infection control & $4 / 12(33.3)$ \\
HIV status of source patient already & \\
$\quad$ known or determined after exposure & $4 / 12(33.3)$ \\
HCW tested for HIV after exposure & $3 / 11(27.3)$ \\
HBV infection status of source patient & \\
$\quad$ already known or determined after & \\
$\quad$ exposure & $0 / 12$ \\
HCW tested for HBV after exposure & $1 / 12(8.3)$ \\
\hline
\end{tabular}

NOTE. Because of rounding, percentages may not add up to $100 . \mathrm{HBV}$, hepatitis B virus; HIV, human immunodeficiency virus.

a The most recent sharps injury was the injury closest in time to the interview; there were 12 such exposures.

b Percentages for sharps injuries are based on 12 respondents' most recent exposures.

' Percentages for outcomes are based on 12 respondents' most recent exposures. d Data are missing on HIV testing for $1 \mathrm{HCW}$. 


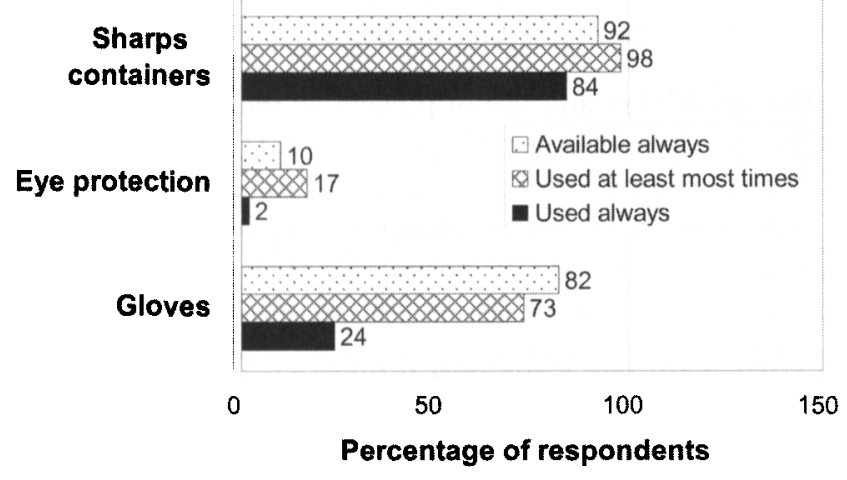

FIGURE. Percentage of respondents who reported that protective devices were always available and those who reported that they always or at least most times used those protective devices when appropriate for the type of work they were performing.

Tanzania showing a high but underreported incidence of needlestick injuries. ${ }^{4}$ A recent World Health Organization study reported that half of the estimated $16,000 \mathrm{HCV}$ infections, $66,000 \mathrm{HBV}$ infections, and approximately 1,000 HIV infections that occur annually in HCWs are attributable to sharps injuries. ${ }^{5}$

The Kenyan HCWs surveyed had significant concern regarding their occupational risk of HIV acquisition. HIV prevalence was perceived to be high among patients at their workplace, and many HCWs seemed to have an inflated perception of the risk of HIV transmission via needlestick injury. By contrast, respondents' concerns about $\mathrm{HBV}$ and $\mathrm{HCV}$ infection appeared to be less intense but were still present. A recent national survey in Kenya estimated HIV prevalence among rural residents at 5.6\%. ${ }^{6}$ In a previous survey of outpatients in 3 hospitals in Eastern Kenya, 11.4\% were documented to test positive for HBV surface antigen. ${ }^{7}$ In neighboring Tanzania, seroprevalence of $\mathrm{HCV}$ infection in the general population was estimated to be $1.4 \% .{ }^{8}$ Even if HCWs' estimates of occupational risks for infection by bloodborne pathogens are inflated, their perceptions suggest an added emotional stress for HCWs working in a resource-limited healthcare system.

Measures to prevent occupational exposures to bloodborne pathogens exist in this mission hospital, but are in need of further support and development. In 1995, Maua Methodist Hospital had increased provision of sharps disposal boxes and gloves. HCWs were instructed to report contaminated sharps injuries and/or body fluid splashes to mucosa. HBV vaccination was offered free of charge to HCWs, although vaccine was not always available. At the time of this study, two-thirds of the HCWs surveyed had received $\mathrm{HBV}$ vaccine, but most reported receiving an incomplete vaccination series. The importance of $\mathrm{HBV}$ vaccination for all at-risk HCWs was highlighted at a recent meeting of the Safe Injection Global Network in 2005. ${ }^{8}$ It is encouraging that almost all of our respondents found that sharps disposal containers were al- ways available when needed. However, though gloves were frequently available, glove use was reported to be less uniform.

Underreporting of injuries is one barrier to adequate assessment by hospital support staff. However, this study was conducted when availability of postexposure prophylaxis (ie, antiretroviral drugs for HIV exposure and immunoglobulin for HBV exposure) was extremely limited, likely serving as a major disincentive for HCWs to report injuries and undergo a formal risk review. Fortunately, recent funding has enabled initiation of a postexposure prophylaxis program for HIV at Maua Methodist Hospital, offering new hope to HCWs and lending urgency to reporting.

We acknowledge some limitations to our results. First, the generalizability of these data is limited because they are based on a small convenience sample of mission-hospital HCWs. However our findings are consistent with previous studies regarding risks of bloodborne pathogen transmission in countries with limited resources. ${ }^{4,9,10}$ Second, generalization to urban or public hospitals is not possible because of differences between those institutions and rural mission hospitals. Lastly, the study was based on self-reported occupational exposures and prevention practices. Even so, our conclusions are less subject to recall bias because they were focused on the most recent sharps injuries-stressful, memorable events.

In summary, we documented HCW' concerns about and exposure to bloodborne pathogens in a rural Kenyan setting where HIV and viral hepatitis may be prevalent. Although there is a need for improvement, some measures are being taken to prevent and respond to occupational exposure to bloodborne pathogens. To support much-needed occupational safety among HCWs in rural Kenya, it is hoped that coverage for HBV vaccination will be expanded, access to sharps safety devices will be increased, and postexposure prophylaxis will be offered for HIV exposure. So that hospitals are not operating in isolation in regard to this important public health activity, there is a need for national campaigns to address cultural perceptions leading to the overuse of injections and to support broader training for and implementation of occupational safety measures to protect HCWs against bloodborne pathogens.

\section{ACKNOWLEDGMENTS}

We thank Dr. Denise Cardo, Centers for Disease Control and Prevention; Dr. Le Thi Anh Thu, Cho Ray Hospital, Ho Chi Minh City, Vietnam; and Dr. Annette Sohn, University of California San Francisco, for providing the original survey instrument. We also thank Maua Methodist Hospital healthcare workers for their participation in the study.

Potential conflicts of interest. D.N.F. reports that he has served as a paid consultant to Surutek. N.M.M., S.G.I., and K.G.J. report no conflicts of interest relevant to this article.

Nkuchia M. M'ikanatha, DrPH, MPH; Stanley G. Imunya, MPH; David N. Fisman, MD, MPH; Kathleen G. Julian, MD 
From the Pennsylvania Department of Health, Harrisburg (N.M.M.), the Center for Clinical Epidemiology and Biostatistics, University of Pennsylvania School of Medicine, Philadelphia (N.M.M.), and the Division of Infectious Diseases, Penn State Milton S. Hershey Medical Center, Pennsylvania State University, Hershey (K.G.J.), Pennsylvania; the Center for Health and Wellbeing, Princeton University, Princeton, New Jersey (D.N.F.); and the Department of Community Health, Maua Methodist Hospital, Kenya (S.G.I.).

Address reprint requests to Nkuchia M. M'ikanatha, DrPH, MPH, Division of Infectious Disease Epidemiology, Pennsylvania Department of Health, Health and Welfare Building, Harrisburg, PA 17108 (nmikanatha@state.pa.us).

Infect Control Hosp Epidemiol 2007; 28:761-763

(C) 2007 by The Society for Healthcare Epidemiology of America. All rights reserved. 0899-823X/2007/2806-0027\$15.00. DOI: $10.1086 / 519699$

\section{REFERENCES}

1. Panlilio AL, Cardo DM, Grohskopf LA, Heneine W, Ross CS; U.S. Public Health Service. Updated U.S. Public Health Service guidelines for the management of occupational exposures to HIV and recommendations for postexposure prophylaxis. MMWR Recomm Rep 2005; 54:1-17.

2. Safety of injections in immunization programmes: WHO recommended policy. Geneva: World Health Organization; 1998. Available at http:// www.who.int/vaccines-documents/DoxGen/h5-inj.htm. Accessed April $17,2006$.

3. Sagoe-Moses C, Pearson RD, Perry J, Jagger J. Risks to health care workers in developing countries. $N$ Engl J Med 2001; 345:538-541.

4. Gumodoka B, Favot I, Berege ZA, Dolmans WM. Occupational exposure to the risk of HIV infection among health care workers in Mwanza Region, United Republic of Tanzania. Bull World Health Organ 1997; 75:133-140.

5. Prüss-Üstün A, Rapiti E, Hutin Y. Sharps Injuries: Global Burden of Disease From Sharps Injuries to Health-Care Workers. (WHO Environmental Burden of Disease Series, No. 3). Geneva: World Health Organization; 2003.

6. Central Bureau of Statistics (CBS) [Kenya], Ministry of Health (MOH), and ORC Macro. Kenya Demographic and Health Survey. Calverton, Maryland: NCPD, MOH, and ORC Macro; 2003.

7. Hyams KC, Okoth FA, Tukei PM, et al. Epidemiology of hepatitis B in eastern Kenya. J Med Virol 1989; 28:106-109.

8. Tess BH, Levin A, Brubaker G, et al. Seroprevalence of hepatitis $C$ virus in the general population of northwest Tanzania. Am J Trop Med Hyg $2000 ; 62: 138-141$.

9. World Health Organization. Safe Injection Global Network (SIGN). Report of the Global Injection Safety and Infection Control meeting, 14-16 November, 2005. Geneva, World Health Organization, 2006. Available at: http: //www.who.int/injection_safety/Final-SIGNHanoiReport22March06.pdf. Accessed July 15, 2006.

10. Tarantola A, Koumare A, Rachline A, et al. A descriptive, retrospective study of 567 accidental blood exposures in healthcare workers in three West African countries. J Hosp Infect 2005; 60:276-282.

\section{Pseudo-outbreak of Ochrobactrum anthropi Bacteremia Related to Cross-Contamination From Erythrocyte Sedimentation Tubes}

To the Editor-Blood cultures have an important role in clinical practice, but it is essential to be able to distinguish between true bacteremia and contamination resulting from inadequate aseptic technique, particularly when unusual microorganisms are recovered. We describe a pseudo-outbreak of this kind at our hospital. Hospital Universidad Católica is a 500-bed, tertiary care teaching hospital in Santiago, Chile. Our health network also includes 13 outpatient clinics where specimens are collected, which are sent to a central clinical microbiology laboratory. During a 4 month period, 8 patients from the hospital and outpatient clinics had Ochrobactrum anthropi isolated from blood cultures (Table).

$O$. anthropi is a gram-negative bacillus that can be found in the environment, including in plants and water sources.' It is considered to be of low pathogenicity, and most of the reported infections occur in individuals with intravascular devices or impaired immunity. ${ }^{2,3}$ Reported infections have included pacemaker lead-associated infection, endocarditis, postoperative endophtalmitis, necrotizing fasciitis, and osteochondritis of the foot after a wound. O. anthropi bacteremia has also been linked to contaminated infusates. ${ }^{4}$

The above-mentioned 8 cases represented a dramatic increase in the number of blood cultures positive for $O$. anthropi at our institution, and an outbreak investigation was initiated. A case patient was defined as any patient from whom $O$. anthropi was isolated in blood culture from January through April 2000 (ie, during the outbreak period). Case patients were identified at the university health network by microbiology reports and infection control surveillance. Rates of $O$. anthropi isolation for the outbreak period and the preoutbreak period (January 1998 to January 2000) were compared. Clinical information was collected by medical records review and health personnel interviews. Current practice and writ-

TABLE. Clinical Characteristics of 8 Case Patients Involved in a Pseudo-outbreak of Ochrobactrum anthropi Bacteremia

\begin{tabular}{lc}
\hline Characteristics & $\begin{array}{c}\text { Case patients } \\
(N=8)\end{array}$ \\
\hline Male sex & $5(63)$ \\
Age, median (range), years & $40(4-79)$ \\
Clinical diagnosis & \\
Febrile syndrome & $4(50)$ \\
Pneumonia & $2(25)$ \\
Other & $2(25)$ \\
Clinical ward & \\
Internal medicine & $4(50)$ \\
Outpatient clinic 1 & $1(13)$ \\
Outpatient clinic 2 & $1(13)$ \\
Outpatient clinic 3 & $1(13)$ \\
Outpatient clinic 4 & $1(13)$ \\
Proportion of blood cultures positive & \\
$1 / 1$ & $3(37)$ \\
$1 / 2$ & $3(37)$ \\
$1 / 3$ & $1(13)$ \\
$1 / 4$ & $1(13)$ \\
Polymicrobial culture results & $1(13)$ \\
Time to positive culture results, median (range), h & $29(21-35)$ \\
Isolates clinically considered contaminants & $8(100)$ \\
\hline
\end{tabular}

NOTE. Data are no. (\%) of patients, unless otherwise indicated.

a No. of blood cultures positive for $O$. anthropi/ total no. of blood culture bottles inoculated. 